\title{
A review of nonlinear applications in silicon optical fibers from telecom wavelengths into the mid-infrared spectral region
}

\author{
Li Shen ${ }^{\mathrm{a}, \mathrm{b}, *}$, Haonan Ren ${ }^{\mathrm{a}}$, Meng Huang ${ }^{\mathrm{a}}$, Dong Wu${ }^{\mathrm{a}}$, and Anna C. Peacock ${ }^{\mathrm{a}}$ \\ ${ }^{\text {a } O p t o e l e c t r o n i c s ~ R e s e a r c h ~ C e n t r e, ~ U n i v e r s i t y ~ o f ~ S o u t h a m p t o n, ~ S o u t h a m p t o n, ~ S O 17 ~}$ \\ 1BJ, UK \\ ${ }^{\mathrm{b}}$ Wuhan National Laboratory for Optoelectronics (WNLO), Huazhong University of \\ Science and Technology, Wuhan, 430074, China
}

\begin{abstract}
Glass-glad silicon core fibers are attracting considerable attention for use in nonlinear applications due to their tight light confinement and intrinsically high third-order nonlinearities. Recent advances in the fabrication procedures have shown that molten core fiber drawing and post-processing techniques can be combined in a versatile approach to produce low-loss fibers with submicron-sized crystalline cores. In this paper, we review current progress in the nonlinear application of silicon-based optical fibers from telecom wavelengths into the mid-infrared. Particular attention is paid to dispersion engineering the fibers to achieve efficient parametric amplification, wavelength conversion and midinfrared supercontinuum generation.
\end{abstract}

Keywords: Fiber optics; fiber nonlinear optics; supercontinuum generation; silicon photonics

\footnotetext{
* Corresponding author. Tel.: +44 (0)23 80593155; fax: +44 (0)23 80593142. E-mail address: lishen@hust.edu.cn
} 


\section{Introduction}

Silicon is an excellent nonlinear material, possessing a high third-order susceptibility, and there are various nonlinear effects that can be used to generate and process optical signals across a broad wavelength regime, spanning the telecom band up to the midinfrared (mid-IR) [1-4]. To date, most demonstrations of nonlinear silicon processing have been realized using the planar silicon-on-insulator (SOI) platform, owing to its compatibility with mature CMOS technologies [5]. Recently, low-loss glass-glad silicon core fibers (SCFs) fabricated via a combination of molten core drawing (MCD) and a fiber tapering post-processing procedure have emerged as an alternative platform, thanks to considerable advancements that have been made in the materials optimization [6-8]. This fabrication method can now routinely produce submicron sized SCFs with optical losses and nonlinear performances that rival their planar SOI waveguide counterparts [9]. Furthermore, these SCFs can be directly spliced to existing optical fiber components, including the pump lasers for nonlinear applications, and thus have the potential for constructing efficient and elegantly packaged all-fiber nonlinear systems [10-13].

It is now ten years since the first nonlinear demonstration in a SCF was reported [14], and nonlinear effects including multiphoton absorption [15], self- and cross-phase modulation (SPM and XPM) [14,16], as well as four-wave mixing (FWM) [17,18] have since been observed, which can be harnessed for use in all-optical modulation, switching, parametric amplification, and supercontinuum generation schemes. It is the aim of this review to present an overview of some of the key achievements obtained using this novel silicon fiber platform for nonlinear applications. Owing to the extended infrared transparency of the silicon core, compared to traditional silica fibers, demonstrations will be presented for wavelengths regions beyond the telecom band and into the mid-IR, where 
there is potential to target applications in free space communications, sensing and healthcare [19].

The remainder of this paper is organized as follows. It begins with a brief discussion in Section 2 of the fabrication and post-processing techniques for SCFs, including the MCD method and thermal tapering process. Section 3 introduces the nonlinear model used to describe light propagation in the SCFs, which is used to characterize nonlinear properties of the silicon core material. Section 4 focuses on the experiments to investigate wavelength conversion and parametric application at telecom wavelengths. Mid-IR supercontinuum generation in the SCFs is presented in detail in Section 5. Section 6 provides discussions and perspectives on opportunities of using these fibers for all-fiber nonlinear systems. Lastly, we conclude the paper.

\section{Silicon core fibers fabrication and post-processing}

It was in 2006 that the first SCF was fabricated by depositing silicon inside a silica capillary via a high pressure chemical vapour deposition (HPCVD) technique [20]. Following this, a complementary fabrication technique was established based on a traditional drawing tower approach, which was termed the MCD method as the silicon core is molten at the temperatures where the silica cladding softens for the drawing process [21]. Over the years, the HPCVD method has proven to be relatively time consuming, with the deposited fibers being limited to lengths of a few centimeters. However, the MCD method is fast, can produce long lengths of SCF and is fully compatible with standard fiber process procedures. Moreover, a post-draw tapering procedure has been developed to further improve the MCD fiber after the drawing to produce crystalline SCFs with micro/nanometer-sized core diameters and relatively low optical losses, which is highly desirable for nonlinear applications [8]. Therefore, 
currently the MCD method is the preferred method for SCF production and will form the focus of this review.

(a)

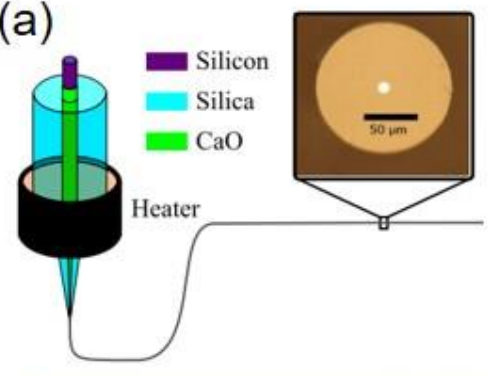

(c) (b) Core $\quad$ Cladding 1 Protection sleeve

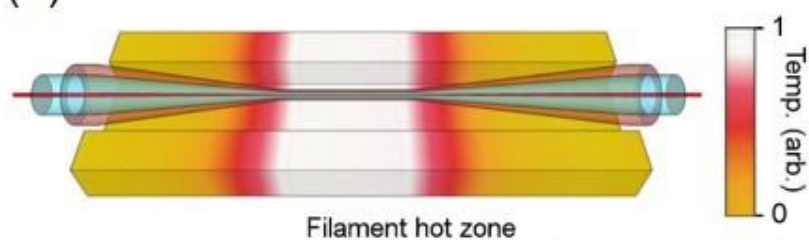

Filament hot zone

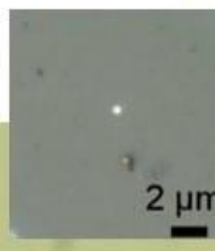

$100 \mu \mathrm{m}$

Fig. 1. (a) Fabrication process of $\mathrm{MCD}$ with $\mathrm{CaO}$ interface modifier and a microscope image of an as-drawn SCF cross-section shown in the inset. (b) Schematic of the tapering process. (c) Microscope image showing tapered silicon core fiber. Inset, microscope image of a submicron core silicon fiber.

Fig. 1(a) shows a schematic of the MCD process, which was first demonstrated by the Ballato Group at Clemson University in 2008 [6]. The preform used to fabricate a SCF is typically made from a silica tube with a silicon rod or powder in the middle. More recently, Nordstrand et al. developed a method to place an interfacial modifier (for example calcium oxide) between the core and cladding, which is used to reduce oxide contaminations from the core and also to reduce the effective expansion mismatch between the materials that can led to cracking [7]. Thus the use of interface layers ultimately helps to achieve smaller drawn SCF core sizes. Typically, the thin calcium oxide layer is formed by pumping a calcium hydroxide solution through the silica tube before drying overnight. Then the SCFs are drawn from the preform at a tempertaure above both the glass transition temperature of the cladding and the melting temperature 
of the silicon core. This technique can produce tens of meters of SCFs with outer diameters $\sim 150 \mu \mathrm{m}$ and core diameters typically around $10 \mu \mathrm{m}$, or larger, as shown by the micoscope image of a SCF facet in Fig. 1(a).

One of the key advantages of the SCF platform is that conventional glass fiber postprocessing techniques can be used to optimize the silicon core materials after the drawing process as well as to tune the core/cladding diameters. For standard silica fibers, tapering is widely used to modify the dimensions in order to change the guiding properties of a fiber such as dispersion, nonlinearity and modal behavior $[22,23]$. The tapering process typically uses a filament to heat and soften the glass fiber before it is stretched to alter the dimensions. As the SCFs are clad in glass, this method can be applied to reduce the core size down to smaller dimensions desired for nonlinear applications, as shown in Fig. 1(b). Moreover, the taper shape can be controlled by the tapering temperature and the stretching motion, including pull speed, start and end position. Importantly, this post-processing method has also been shown to further improve the material quality as the core will recrystallize on cooling, resulting an added benefit of loss reduction [9]. For example, the optical transmission losses in the telecom band have been shown to reduce from $12 \mathrm{~dB} / \mathrm{cm}$ in a $10 \mu \mathrm{m}$ diameter as-drawn SCF to $\sim 2.8 \mathrm{~dB} / \mathrm{cm}$ in a submicron tapered core fiber [8, 18]. Fig. 1(c) illustrates a microscope image of a tapered SCF with a nanoscale core. The crystallinity of the silicon core has been improved close to single crystal after this tapering process, which have been assessed using both Raman spectroscopy and X-ray diffraction (XRD) measurements [9].

\section{Nonlinear optical properties of silicon core fibers}

3.1 Nonlinear pulse propagation 
In order to describe pulse propagation in silicon waveguides or fibers, a modified form of the Nonlinear Schrödinger Equation (NLSE) has been widely used [24]:

$$
\frac{\partial A}{\partial z}=\sum_{m \geq 2}^{\infty} \frac{i^{m+1} \beta_{m} \partial^{m} A}{m ! \partial t^{m}}+i \gamma|A|^{2} A-\frac{1}{2}(\alpha+\sigma) A
$$

where $A$ is the pulse envelope. $\beta_{m}$ are the dispersion parameters and in most cases only $\beta_{2}, \beta_{3}$ are considered, which represent the group velocity dispersion (GVD) and the thirdorder dispersion (TOD) parameters, respectively. $\gamma$ is the third order nonlinearity coefficient. Its real part gives rise to the Kerr nonlinear refractive index $n_{2}$ and the imaginary part is related to the two-photon absorption (TPA) parameter $\beta_{\mathrm{TPA}} . \alpha$ is the linear loss. The free carrier coefficient $\sigma$ that contains both free carrier absorption (FCA, $\left.\sigma_{\mathrm{FCA}}\right)$ and free carrier dispersion $(\mathrm{FCD}, \mu)$ is described as $\sigma=\sigma_{\mathrm{FCA}}(1+i \mu) N_{c}$, where $N_{c}$ is the free carrier density. The carrier density is related to the carriers generated from the TPA process and can be determined via the rate equation [24]:

$$
\frac{d N_{c}}{d t}=\frac{\beta_{T P A}}{2 \hbar \omega} I^{2}-\frac{N_{c}}{\tau_{c}},
$$

where $\tau_{c}$ is the carrier lifetime.

Owing to large refractive index contrast, SCFs can provide tight mode comfinement, which enhances the nonlinear effects by several orders of magnitude compared to silica fibers [1]. Nonlinear transmission in SCFs is mainly governed by TPA and Kerr nonlinear refraction. The nonlinear properties of the SCFs can be characterized via analyzing the nonlinear transmission using a short pump pulse as input source. As TPA manifests as an intensity dependent absorption, the $\beta_{\text {TPA }}$ parameter can be determined simply by monitoring the output power as a function of the coupled input power. Subsequently, the 
size of the nonlinear refractive index $\left(n_{2}\right)$ can be estimated by mapping the spectral broadening due to SPM.

3.2 Two-photon absorption and self-phase modulation
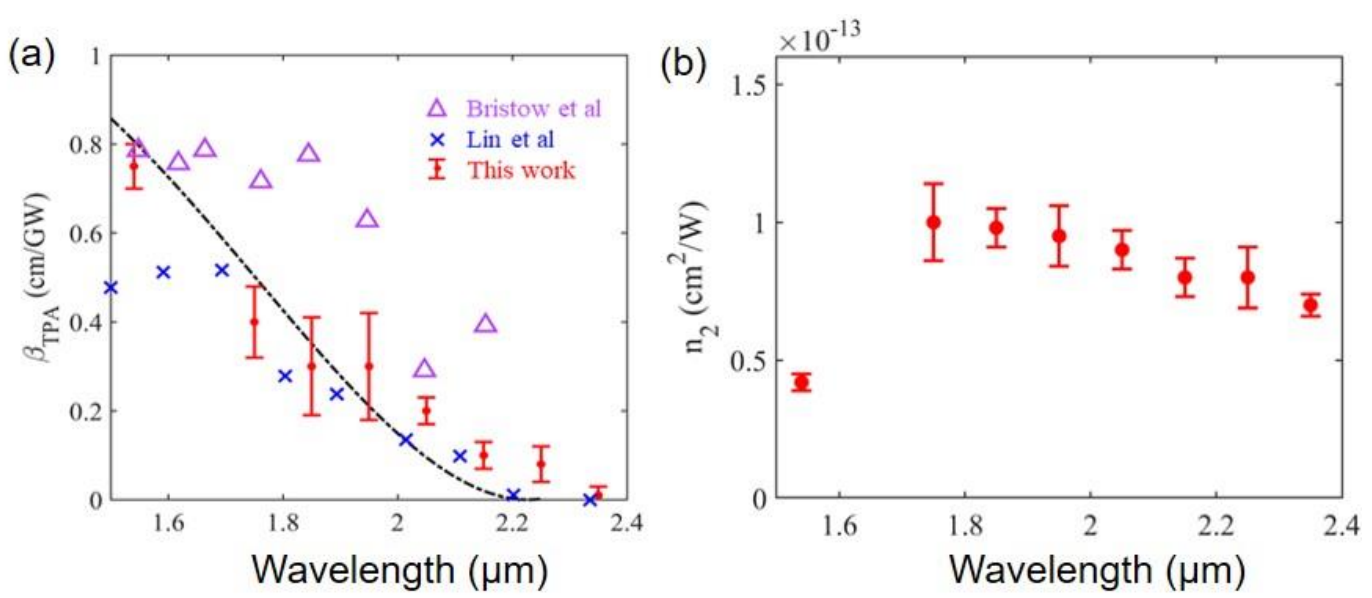

Fig. 2. (a) Measured TPA parameter as a function of wavelength, together with data points from measurements by other groups [27], [28]. Error bars represent the uncertainty in the input powers. Dashed line is a theoretical fit based on calculations of Garcia and Kalyanaraman [29]. (b) Wavelength dependence of the Kerr nonlinear coefficient $n_{2}$. Error bars represent the uncertainty in the input pulse power.

To investigate the suitability of the SCFs for nonlinear propagation at various wavelengths from the telecom band up to the mid-infrared, a set of power dependent transmission measurements were conducted. We used an SCF that was tapered down from an as-drawn fiber (outer cladding diameter of $377 \mu \mathrm{m}$ and a core diameter of $30 \mu \mathrm{m}$ ) to achieve a uniform waist region with a core diameter of $3 \mu \mathrm{m}$ over a $1 \mathrm{~cm}$ length. The linear loss of this SCF measured by the cutback method stays consistently around $2 \mathrm{~dB} / \mathrm{cm}$ over the entire wavelength region from 1.55 to $2.5 \mu \mathrm{m}$ [25].

Characterization of the TPA parameter was performed by measuring the saturation of the output pulses as a function of increasing input power. The TPA coefficients were obtained by comparing the experimental transmission results with numerical fits via 
solving Eqs. (1) and (2), with the remaining material parameters such as the FCA and dispersion estimated from the single crystal values [26]. Fig. 2(a) plots the $\beta_{\text {TPA }}$ as a function of wavelength, with previous measurements of crystal silicon materials from Refs. [27] and [28]. The $\beta_{\mathrm{TPA}}$ drops from $\sim 0.75 \mathrm{~cm} / \mathrm{GW}$ to $\sim 0.1 \mathrm{~cm} / \mathrm{GW}$ from $1.54 \mu \mathrm{m}$ to $2.3 \mu \mathrm{m}$. That is, it becomes negligible after the TPA edge. It can be seen that although there are small differences for the different studies, these measurements follow a similar decreasing trend with increasing wavelength.

The spectral broadening induced by SPM at different power levels was then studied to determine the values of the Kerr coefficient $n_{2}$ over this wavelength range. By comparing experimental spectra with the numercal simulations at both low and high input peak intensities, the value of $n_{2}$ can then be determined [14]. The estimated Kerr coefficient $n_{2}$ for each central wavelength is plotted in Fig. 2(b). The estimated value of $n_{2}$ is $\sim 0.42 \times 10^{-13} \mathrm{~cm}^{2} / \mathrm{GW}$ at $1.54 \mu \mathrm{m}$, which is similar to the value reported for crystal silicon waveguides [26]. As the input pulse wavelength changes from $1.54 \mu \mathrm{m}$ across the TPA edge, the $n_{2}$ value first increases significantly up to a value of $1.0 \times 10^{-13} \mathrm{~cm}^{2} / \mathrm{W}$ at 1.75 $\mu \mathrm{m}$, then it decreases slowly as the wavelength increases, down to a modest value of 0.7 $\times 10^{-13} \mathrm{~cm}^{2} / \mathrm{W}$ at $2.35 \mu \mathrm{m}$. The $n_{2}$ dispersion profile is consistent with the measurements by Bristow et al. [27] and the theoretical predictions of Hon et al. [30], where the values of $n_{2}$ peak at approximately the same wavelength.

\subsection{Nonlinear figure of merit}

The dispersion of the nonlinear figure of merit $\left(\mathrm{FOM}_{\mathrm{NL}}\right)$ of the SCF is then calculated by using the definition $n_{2} / \lambda \beta_{\text {TPA }}$, as shown in the Fig. 3 . It can be clearly seen that, the $\mathrm{FOM}_{\mathrm{NL}}$ increases with increasing wavelength. The FOM $\mathrm{NL}$ in the telecommunications band has the lowest value of $\simeq 0.36$, which limits the nonlinear performance of the SCFs 
in this region. However, this is also a general conclusion for all silicon-based waveguides. The increasing trend of the $\mathrm{FOM}_{\mathrm{NL}}$ suggests that these fibers are more suitable for nonlinear applications for wavelengths toward mid-IR region. At $2.35 \mu \mathrm{m}$, where the TPA disappears, the $\mathrm{FOM}_{\mathrm{NL}}$ is no longer an accurate measure of the nonlinear performance as 3PA becomes the dominant nonlinear absorption effect in this wavelength region. The $\mathrm{FOM}_{\mathrm{NL}}$ is then defined as $n_{2} / \lambda \beta_{3 \mathrm{PA}}$, where $I$ is the light intensity inside the nonlinear medium. Using data for the 3PA parameter of single crystal silicon from the literature [31], the $\mathrm{FOM}_{\mathrm{NL}}$ just beyond the TPA cut-off wavelength is calculated at a typical input intensity $\left(1 \mathrm{GW} / \mathrm{cm}^{2}\right)$ for comparison. This is shown as the square data point in Fig. 3. Even using this definition, the $\mathrm{FOM}_{\mathrm{NL}}$ keeps increasing for longer wavelengths, so the SCFs are expected to have a higher nonlinear performance in this region. However, this should be verified by future work as it is possible that the increasing 3PA values may eventually limit the nonlinear performance.

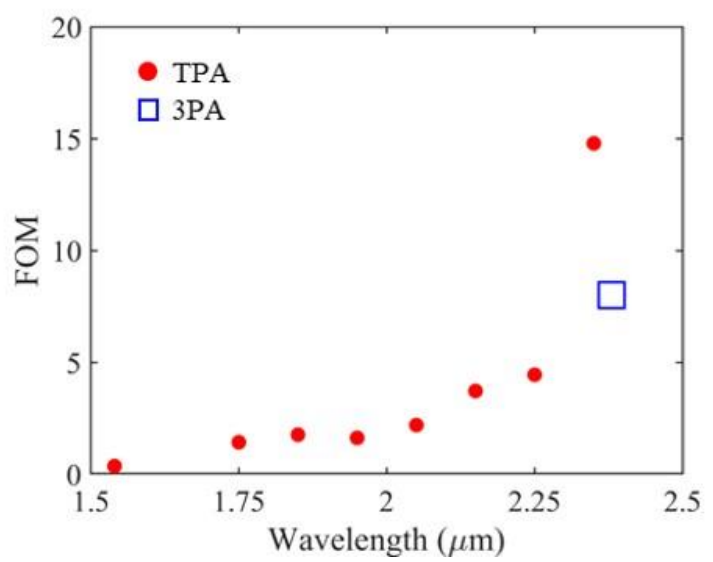

Fig. 3. Wavelength dispersion of the FOM $\mathrm{NL}$.

\section{Wavelength conversion and parametric amplification at telecom wavelengths}

FWM can be used for wavelength conversion and parametric amplification when phase matching conditions are satisfied. In the FWM process, two photons from the pump are converted to a signal and an idler photon. If the high power pump is combined with a 
signal beam, this will result in optical amplification of the signal and the generation of the converted idler beam [3]. In general, efficient phase-matching occurs when the pump wavelength is located in the anomalous dispersion regime, or is close to the zero dispersion wavelength (ZDW) [32]. The nonlinear characterization presented in section 3 was conducted using a SCF with large core diameter $(\sim 3000 \mathrm{~nm})$, exhibiting large normal material dispersion at telecom wavelengths, as shown in Fig. 4(a). In order to observe efficient FWM in SCFs, a much smaller core diameter (usually $<1 \mu \mathrm{m}$ ) is required to access anomalous dispersion in the telecom band.
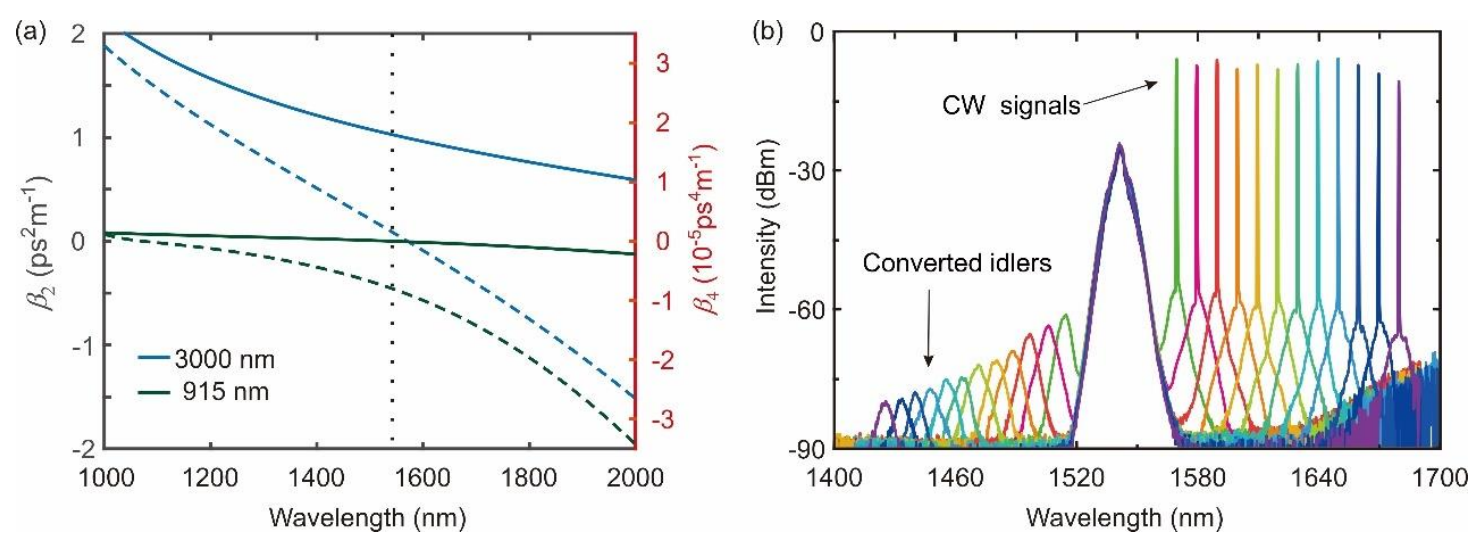

Fig. 4. (a) Calculated GVD (solid lines) and FOD (dashed) as functions of wavelength for the SCF with different core diameters shown in the legend. The vertical dash line indicates the pump wavelength. (b) Transmission spectra taken at the output of the SCF as the signal wavelength is tuned from 1570 to $1680 \mathrm{~nm}$.

A significant breakthrough was made when low-loss submicron SCFs were produced using a two-step tapering procedure [18]. A SCF with a constant submicron waist (915 $\mathrm{nm}$ ) was fabricated over a length of $\sim 5 \mathrm{~mm}$ by applying two sets of optimized parameters (e.g., the filament power and the pulling velocity), firstly to achieve a micron-sized core and then a submicron core. To facilitate efficient coupling, the tapered micron-sized transition regions (maximum diameter of $4 \mu \mathrm{m}$ ) were retained at each end of the fiber. The fiber used in this work was $8 \mathrm{~mm}$ long including the input and output coupling tapers. 
The propagation loss of the SCF at the $1541 \mathrm{~nm}$ pump wavelength was estimated to be $\sim 2.8 \mathrm{~dB} / \mathrm{cm}$, which represents the lowest value reported for a submicron SCF. The nonlinearity was enhanced by the reduction in the mode area in the submicron waist. More importantly, the increased negative waveguide dispersion could compensate for the material dispersion to access the anomalous dispersion regime [33], as illustrated in Fig. 4(a). As the pump wavelength is close to the ZDW of this SCF, fourth order dispersion (FOD,$\left.\beta_{4}\right)$ must be taken into account. Thus, the phase-matching condition can be written as: $2 \gamma P_{\text {pump }}=-\beta_{2} \Delta \omega^{2}-\frac{1}{12} \beta_{4} \Delta \omega^{4}$, where $\Delta \omega$ is the frequency difference between the pump and signal. To demonstrate wavelength conversion and parametric amplification, the tapered submicron SCF was pumped with a mode-locked femtosecond laser (full width at half maximum $T_{\mathrm{FWHM}} \sim 670 \mathrm{fs}$ ) at $1541 \mathrm{~nm}$. At this pump wavelength, the SCF exhibits a very small positive GVD $\left(\beta_{2} \sim 0.038 \mathrm{ps}^{2} / \mathrm{m}\right)$ and a negative FOD $\left(\beta_{4} \sim-0.8\right.$ $\times 10^{-5} \mathrm{ps}^{4} / \mathrm{m}$ ), which permits broadband phase-matching over a conversion bandwidth of $\sim 380 \mathrm{~nm}$.

To investigate parametric amplification, a tunable CW laser was used as a signal beam. By tuning the low power CW signal from 1570 to $1680 \mathrm{~nm}$ (limited by the tuning range of our source), the signal can be down-converted by 260 nm (32 THz), from $1680 \mathrm{~nm}$ to $1420 \mathrm{~nm}$, covering the entire S-, C-, and L- telecom bands. The overlaid FWM spectra are plotted in Fig. 4(b). Owing to the use of a pulsed pump, the amplified signals and generated idlers also occur as a train of short pulses, which is evident from their broad bandwidths. The on/off parametric signal gain and the idler conversion gain can be extracted by converting the time-averaged power measured by the OSA to the peak power using a weighted duty cycle factor $F=1 /(40 \mathrm{MHz} \cdot 670 \mathrm{fs})$. Detailed methods can be found in Refs. $[34,35]$. The extracted values of the parametric signal gain and idler 
conversion gain reveal that a large maximum on/off signal gain of $\sim 9 \mathrm{~dB}$ is achieved at a wavelength of $1570 \mathrm{~nm}$, with a corresponding idler conversion gain of $\sim 8.5 \mathrm{~dB}$. Such a large on/off parametric signal gain in our tapered SCF is sufficient to overcome the moderate fiber insertion losses $(7 \mathrm{~dB})$, demonstrating a $2 \mathrm{~dB}$ net off-waveguide gain. To the best of our knowledge, this is the first time net off-waveguide gain has been demonstrated in a crystalline silicon waveguide using a telecom pump, and is certainly the first demonstration in a polysilicon waveguide. Moreover, the maximum signal gain obtained in our $5 \mathrm{~mm}$ long SCF exhibits more than a $3 \mathrm{~dB}$ improvement over the current highest record in a $17 \mathrm{~mm}$ nanophotonic silicon waveguide in the telecom band, which was limited by strong TPA induced saturation [3].

\section{Mid-infrared supercontinuum generation}

5.1 Supercontinuum generation in a straight SCF

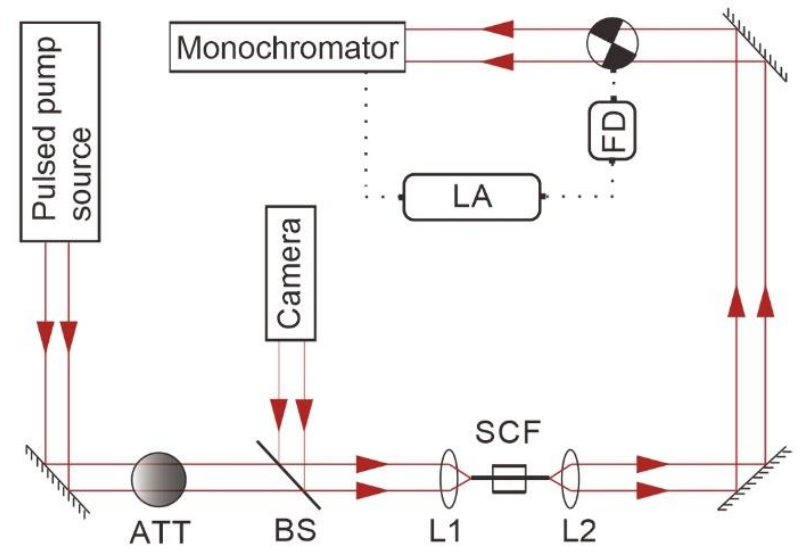

Fig. 5. Experimental setup of supercontinuum generation in a tapered SCF. The pulsed pump source is coupled into the tapered SCF with a lens (L1). The output light is collected with a second lens (L2) and measured by a monochromator via free space alignment. Beam splitters (BS) are used to direct light at the fiber input onto a CCD camera to monitor the coupling. ATT, attenuator. LA, lock-in amplifier. FD, fan driver. 
The MCD technique and post tapering process can provide low-loss SCFs with various core sizes from submicron to a few microns in diameter. Thanks to their high nonlinearity, these SCFs have opened a route for the generation of broadband supercontinuum at relatively modest pump powers. Firstly, to investigate supercontinuum generation in the same SCF used in Section 3, the experimental setup shown in Fig. 5 was used with a pump wavelength at $2.4 \mu \mathrm{m}$, where the TPA is negligible. The pulsed pump was generated using an optical parametric oscillator (OPO), and had a duration of $~ 100 \mathrm{fs}$ (FWHM) and a $80 \mathrm{MHz}$ repetition rate, and, at this wavelength, was positioned in the normal dispersion regime. The beam was launched into the SCF via lens L1. To help optimize the coupling into the fundamental mode, the reflection from the fiber input facet was collected by L1 and guided by a beam splitter (BS) to a CCD camera to monitor the input injection. The output light was collected by another lens L2 and guided into a monochromator (Bentham TMc300) to record the spectrum. An attenuator was used to control the pump power and the signal from the monochromator was detected by a lock-in amplifier (LA) with a fan driver (FD).

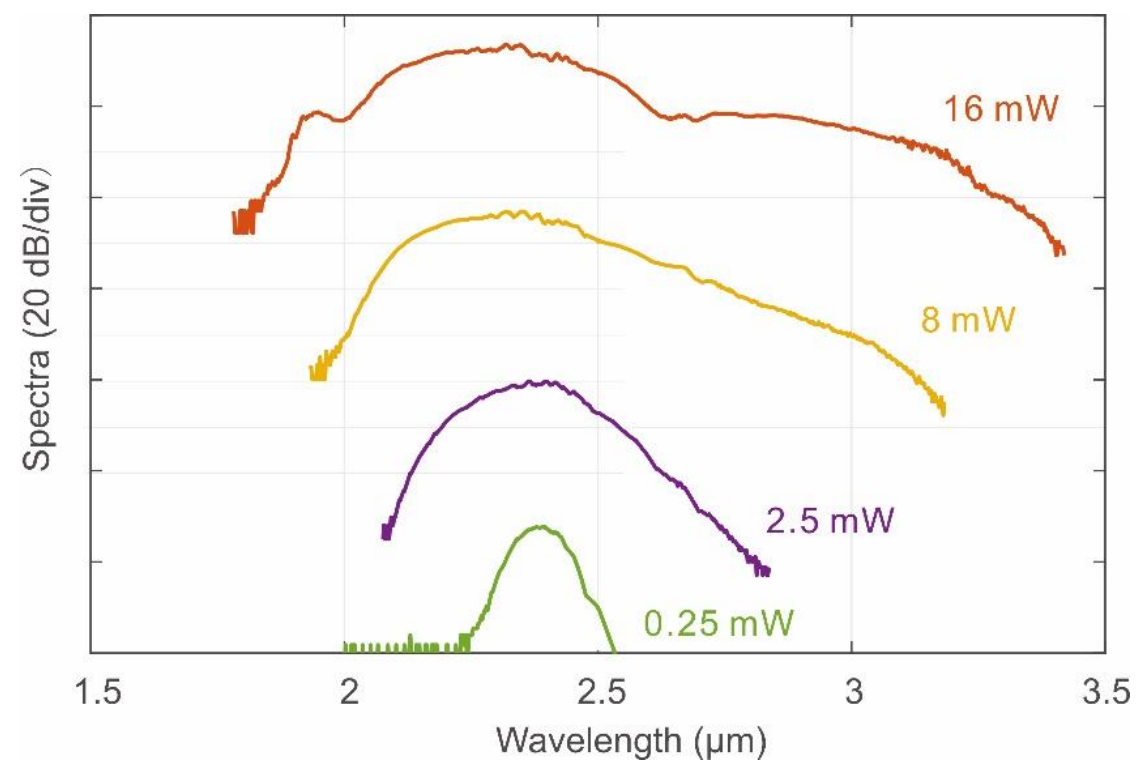

Fig. 6. Supercontinuum generation in a straight $\mathrm{SCF}(3 \mu \mathrm{m})$ pumped at $2.4 \mu \mathrm{m}$. 
The output supercontinuum spectral evolution is shown in Fig. 6, as the coupled average pump powers increase from 0.25 to $16 \mathrm{~mW}$. The maximum generated spectrum is from 1.82 to $3.34 \mu \mathrm{m}$ at the $-40 \mathrm{~dB}$ level when the coupled pump power is $16 \mathrm{~mW}$. This corresponds to $\sim 0.9$ of an octave and represents the broadest spectrum generated in a crystalline silicon waveguide pumped in the normal dispersion regime. A simple way to increase the spectral broadening even further would be to pump the SCF in the anomalous dispersion regime, either by using a longer wavelength pump or by further reducing the core size. However, the coupling efficiency will become lower if SCFs with smaller diameters were used. The low coupling efficiency will ultimately restrict the output supercontinuum power up to a few microwatts [17]. Although this supercontinuum exhibits a modest bandwidth, its flatness and coherence are better than continuum spectra pumped in the anomalous dispersion region because SPM dominates the broadening mechanism. Thus supercontinuum spectra pumped in the normal dispersion regime are often favoured for applications in spectroscopy and metrology [36].

\subsection{Supercontinuum generation in tapered SCFs}

To improve the supercontinuum generation in the SCFs, we designed an asymmetric taper profile. This profile was chosen as the large input core allowed for a high coupling efficiency, before the core was tapered down slowly to ensure tight confinement in the waist (with minimum mode coupling and radiation loss). The tapered waist had a diameter that positioned the pump in the anomalous dispersion region and a length that was long enough to induce a significant nonlinear phase shift. The sharp up-taper was then employed at the output to reduce the interaction with the lossy cladding and maintain a high collection efficiency for all generated wavelengths. The fiber profile is shown in Fig. 7. Its $\mathrm{ZDW}$ is also shown in the same figure with the dashed purple line indicating the 
location of the pump wavelength. The ZDW keeps shifting along the fiber, which helps to generate broader and flatter supercontinuum spectra, as suggested by previous work undertaken on the planar silicon waveguides $[37,38]$. The fiber core diameter changes from $4 \mu \mathrm{m}$ to $1.5 \mu \mathrm{m}$ and back to $7 \mu \mathrm{m}$ at the output. At the designed pump wavelength of $2.4 \mu \mathrm{m}$, the first $3 \mathrm{~mm}$ of the tapered fiber is in the normal dispersion region; from 3 $\mathrm{mm}$ to $10.5 \mathrm{~mm}$ it is in the anomalous dispersion region; and the last $4 \mathrm{~mm}$ of the tapered fiber is again in the normal dispersion region.

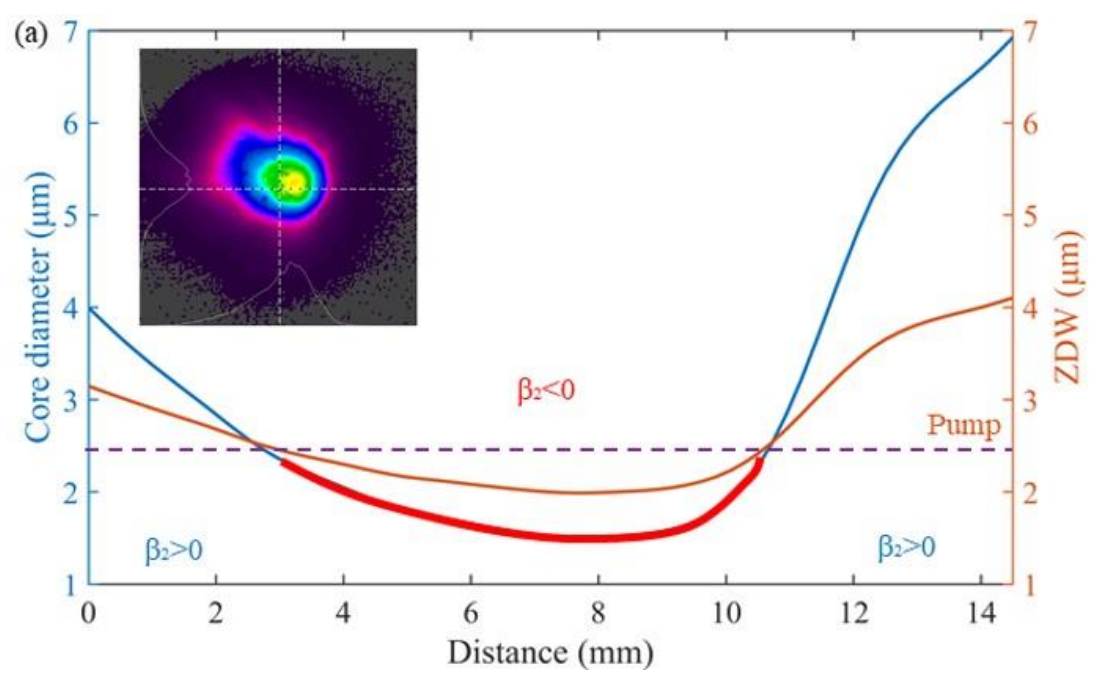

Fig. 7. Tapered fiber profile with the corresponding ZDW when the pump wavelength is at $2.4 \mu \mathrm{m}$. Inset, The beam profile from a mid-IR camera (Pyrocam IIIHR, Ophir Optronics).

The experiment setup has been described in Section 5.1. The alignment was optimised for fundamental mode coupling and the output mode profile is shown in the inset of Fig. 7. The supercontinuum spectra are shown in Fig. 8(a). The broadest spectrum spans from 1.35 to $3 \mu \mathrm{m}$ with only $4 \mathrm{~mW}$ coupled average power. It is much wider than the results in the previous section using a straight SCF at similar pump power. When further increasing the pump power, the spectral bandwidth saturates, which could be due to cladding absorption and/or 3PA. As shown in Fig. 8(b), the fiber was then pumped at 
different wavelengths across $2.2-2.5 \mu \mathrm{m}$. The bandwidth of the obtained spectra are very similar, with only a small change in the spectral shape. Generally speaking, the spectra are flatter but narrower when pumped with shorter wavelengths, as a longer section of the fiber will be in the normal dispersion region and the spectrum will be more influenced by SPM. The spectra pumped at longer wavelengths are wider but less flat, as the pump will be primarily in the anomalous dispersion regime, so that the continuum is dominated by pulse compression and dispersion wave (DW) emission. As the position of the DW is related to the TOD of the fiber, a varying fiber profile can lead to multiple DWs generated at different wavelengths, resulting in an increased bandwidth of the spectrum [39, 40]. This is particularly obvious when pumping the fiber at $2.5 \mu \mathrm{m}$, as shown in Fig. 8(b), where one DW occurs at $1.4 \mu \mathrm{m}$ and another DW occurs at $1.6 \mu \mathrm{m}$.
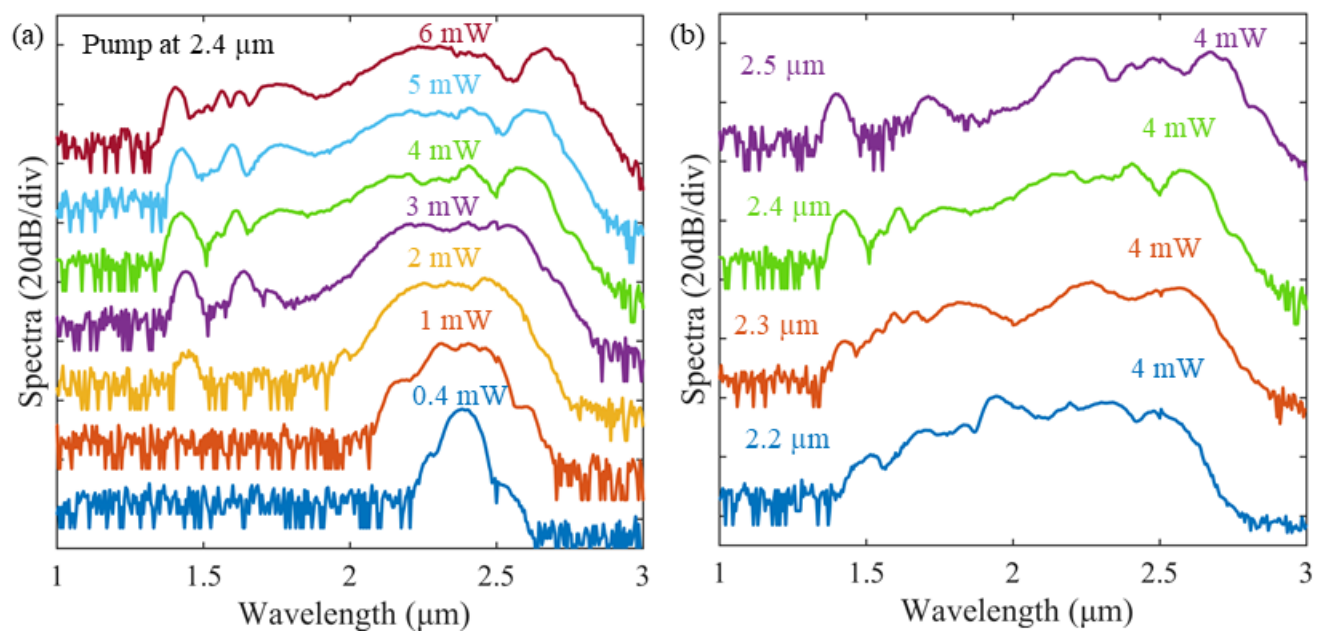

Fig. 8. (a) Supercontinuum spectra generated in the tapered SCF, pumped at $2.4 \mu \mathrm{m}$ with increasing coupled average power. (b) Wavelength tuning of the pump.

The spectral and temporal pulse evolution, as calculated via NLSE simulations, are shown in Fig. 9(a) and (b). It can be seen that the supercontinuum generation process can be divided into three parts. In the first part of the fiber from 0 to $2.5 \mathrm{~mm}$, the pump pulse propagates in the down transition of the taper. In this section, the core size is large and $\beta_{2}$ is positive, the pulse only has some initial spectral broadening through SPM. The peak 
power of the pulse also decreases due to the dispersion and loss. In the second part of the fiber from $2.5 \mathrm{~mm}$ to $11 \mathrm{~mm}$, due to the negative $\beta_{2}$ and smaller fiber core, the pulse compresses and its intensity rises dramatically, which triggers soliton fission and DW emission. In the third part of the fiber, from $11 \mathrm{~mm}$ to the end, the pump enters the normal dispersion again and the light intensity is greatly reduced. As a result, no nonlinear interaction happens in this section and the spectrum remains unchanged.
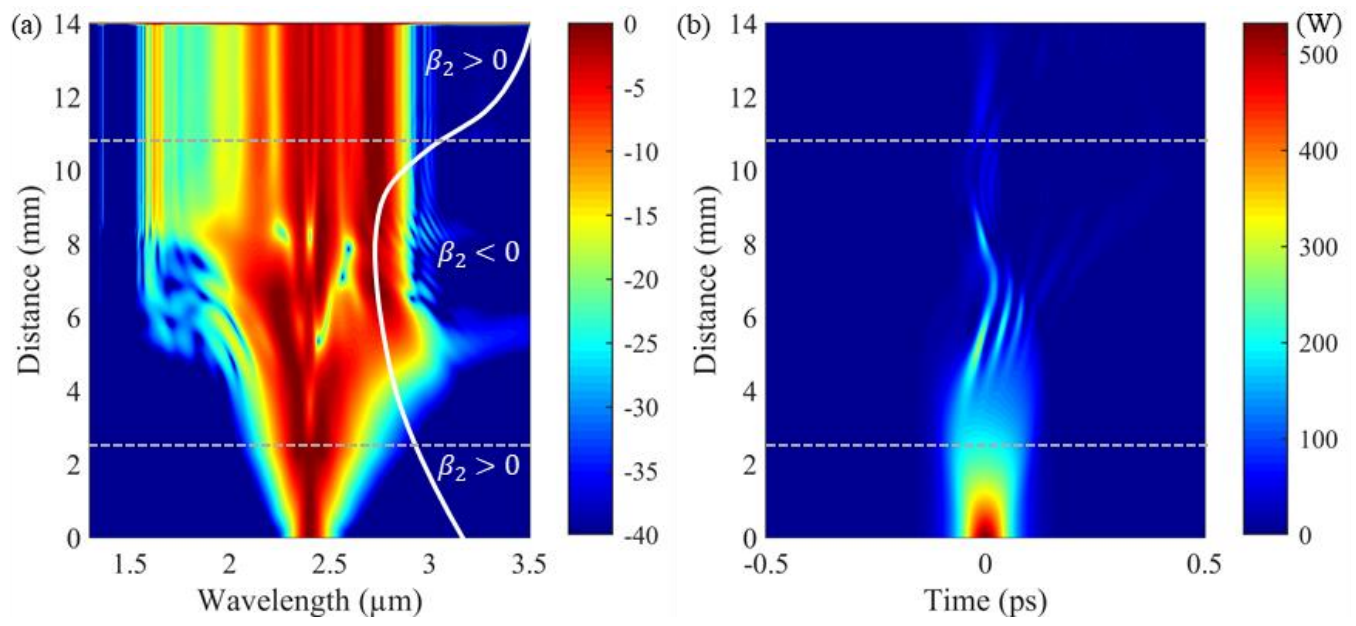

Fig. 9. NLSE simulation of supercontinuum generation in the tapered SCF with $520 \mathrm{~W}$ input pulse peak power. (a) Spectral evolution along the fiber. (b) Pulse propagation along the fiber.

To investigate the wavelength distribution of the supercontinuum in relation to the temporal dynamics, SCG in this fiber was also studied using numerical simulations of the evolution along the fiber length, as shown in Fig. 9(a) and (b). At $z=6 \mathrm{~mm}$, the spectral bandwidth is at the broadest position. The pulse is temporally compressed, and its energy is concentrated. After this point, the supercontinuum gradually stretches temporally due to dispersion, which weakens the frequency mixing, as shown in Fig. 9(b). The output supercontinuum has a $\sim 2$ ps pulse duration. As the broadest position of the spectrum was produced in the middle of the fiber, the fiber section from $6 \mathrm{~mm}$ to $14 \mathrm{~mm}$ does not help 
to extend the spectrum further, but purely introduces additional losses. This indicates that the fiber profile could be further optimised by using a shorter fiber length.

To extend the supercontinuum spectra further into the mid-IR, further optimizations of the taper designs were investigated by taking account the cladding absorption at longer wavelengths, resulting in a tapered SCF with a slightly larger waist diameter and shorter overall length. The profile of the tapered SCF is shown in Fig. 10(a). Simulations show that a very broad supercontinuum spectrum spanning from $2 \mu \mathrm{m}$ to beyond $8 \mu \mathrm{m}$ at the $20 \mathrm{~dB}$ level can be genenated in this optimized tapered SCF when pumped with $3 \mathrm{~kW}$ peak power at a longer pump wavelength $(3 \mu \mathrm{m})$, as illustrated in Fig. 10(b). This simulation indicates that the tapered SCF platform has the potential to produce a supercontinuum spectrum that covers the entire silicon transparency window, even with an absorptive silica cladding [41]. Fabrication of this SCF and experimental measurements are currently ongoing.
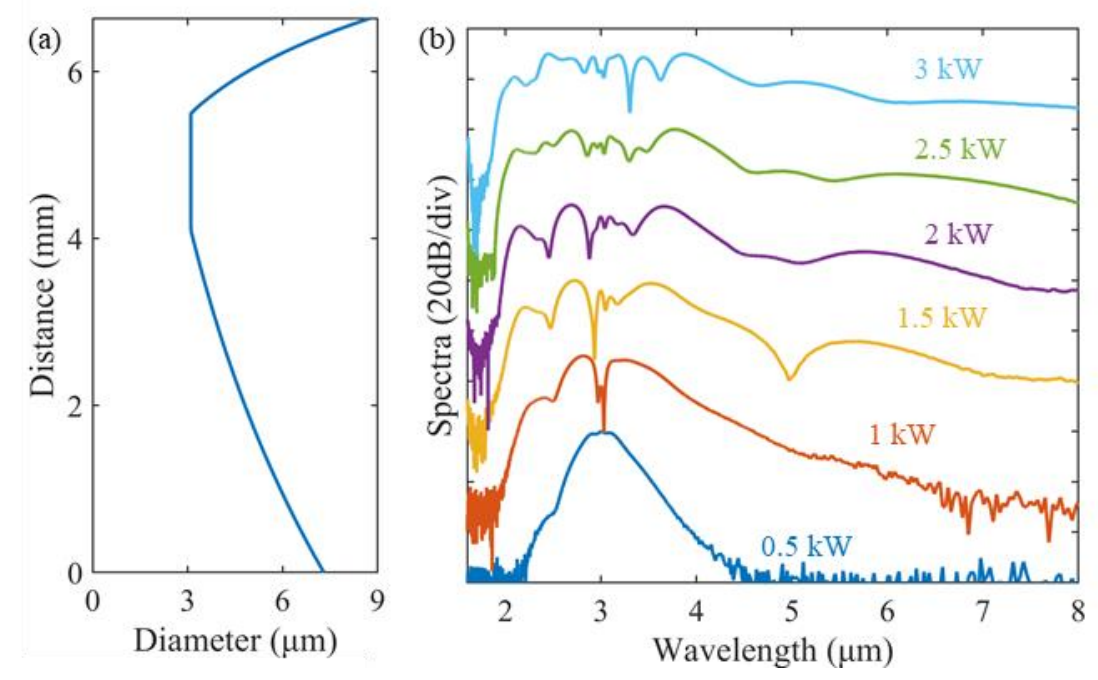

Fig. 10. (a) Optimized tapered SCF profile. (b) Simulated supercontinuum spectra with different coupled peak powers.

\section{Toward all-fiber nonlinear devices}


Coupling light efficiently into and out of the silicon waveguides remains a difficult task due to silicon's high refractive index, which results in significant reflection losses and a large mode mismatch. However, as a key benefit of the SCFs is their compatibility with silica-based optical fibers, we have been able to splice the SCFs directly to standard fibers [10]. An inverse taper coupler has been employed, where we designed and fabricated a nano-spike at the end of the SCF to minimize the reflection losses and better match the optical mode properties of the two different fiber types [42]. Detail of the fabrication processes for our SCFs can be found in Ref. [10].

(a)

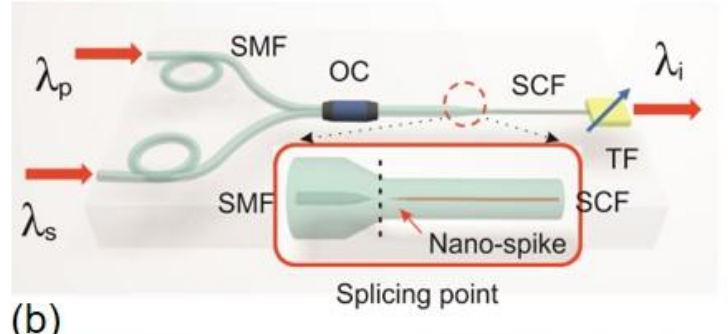

(b)

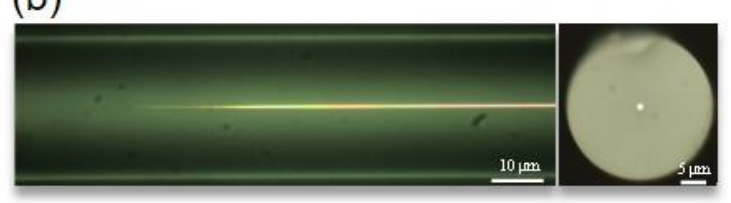

(c)

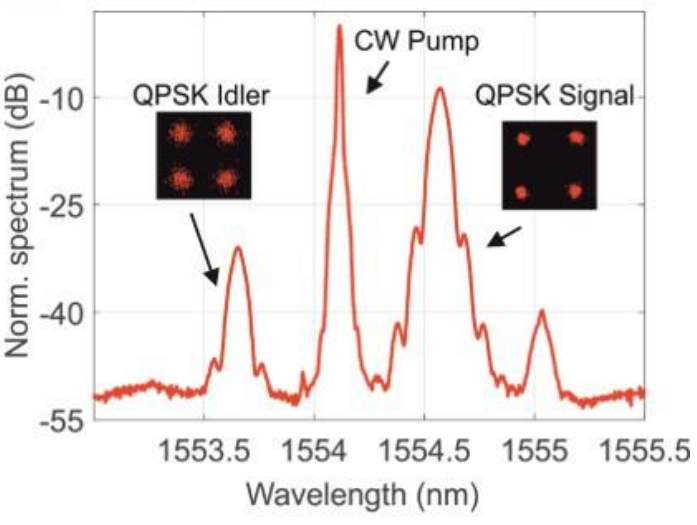

Fig. 11. (a) Schematic of the proposed FWM-based all-fiber wavelength converter. (b) Microscope image of a SCF showing the nano-spike coupling region and the cross-section of the small tapered core. The rainbow colour on the nano-spike indicates the smoothness of its tip. (c) QPSK spectrum of the converted signal at the device output. Signal and idler constellation diagrams are shown as insets.

To demostrate the use of this SCF for all-fiber integrated nonlinear devices, a SCFbased nonlinear wavelength converter (NWC) has been proposed and fabricated [13]. As shown in Fig. 11(a), it consists of a centimeter long SCF with a single mode fiber (SMF) pigtail input coupler. The converter is based on a simple degenerated FWM process, in which two photons from the high power pump wave mix and generate a single signal 
photon and an idler photon at respective wavelengths. Finally, the converted idler is selected out by an external filter. The final fabricated SCF-NWC consists of a $1.1 \mathrm{~cm}$ long SCF with a $200 \mu \mathrm{m}$ long nano-spike, connected to a SMF pigtail with a $4 \mathrm{~mm}$ long tapered transition region to facilitate mode coupling between the two structures. Fig. 11(b) shows microscopy images of the sharp silicon nano-spike and the cleaved end facet of the tapered SCF for output coupling.

The linear propagation loss of the SCF with a $1.1 \mu \mathrm{m}$ core used in the SCF-NWC device was measured to be $2 \mathrm{~dB} / \mathrm{cm}$ at $1550 \mathrm{~nm}$. The coupling efficiency into the SCF section of the NCW was estimated to be $\sim 6.5 \mathrm{~dB}$, which included contributions from the splice loss $\sim 2 \mathrm{~dB}$ and the nano-spike coupling loss $\sim 4.5 \mathrm{~dB}$. This can be further reduced to $<1 \mathrm{~dB}$ by decreasing the silica cladding diameters at the join to $\sim 10 \mu \mathrm{m}$. Using the fabricated SCF-NWC, wavelength conversion of quadrature phase-shift keying (QPSK) signals with a 20-Gb/s bitrate has been achieved. The measured QPSK constellations of the input signal and idler waves are shown in the insets of Fig. 11(c), clearly indicating the modulated data has been successfully converted. The background noise on the weakly converted idler mainly causes performance deterioration.

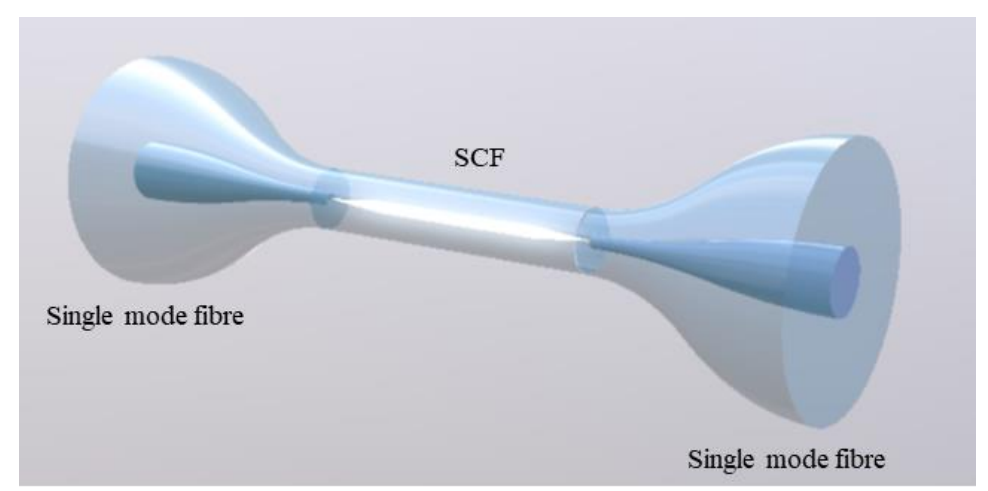

Fig. 12. Concept plan of a SCF device in a standard fiber system.

Although this current SCF-NWC device employs free space output coupling, it could eventually be fully integrated by fabricating nano-spikes on both ends of the SCF. 
However, we are currently restricted from making such a structure due to the lack of a suitable holder to fix the micrometer diameter fiber in the taper rig for the formation of the second spike. Fig. 12 shows a concept plan for a SCF device fully integrated to standard fiber system, which is a promising solution for emerging nonlinear devices designed for optical signal processing applications.

\section{Conclusion}

This paper has reported the recent progress of using SCFs for nonlinear applications from wavelength conversion and supercontinuum generation. The equations governing ultrafast nonlinear pulse propagation in the SCFs have been presented for use in the characterization of the nonlinear properties. Wavelength conversion, optical parametric amplification and octave spanning supercontinuum generation have been demonstrated in the SCFs, with optimized dimensions for either the telecom band or the mid-infrared wavelength regime. Moreover, continued advancements to reduce the losses of SCFs and improve the device coupling efficiency has opened the possibility to fabricate an all-fiberbased wavelength converter for high-speed signal processing applications. We anticipate that these SCFs will provide a versatile platform for many nonlinear applications across the infrared wavelength regime, spanning from next-generation healthcare and communication systems.

\section{Acknowledgements}

L. Shen and H. Ren contributed equally to this paper. The authors acknowledge their colleagues and collaborators who contributed to the cited work, particularly F. Suhailin, Y. Franz, A. F. J. Runge, T. W. Hawkins, J. Ballato, and U. J. Gibson. This publication was supported by the following research funds: the Engineering and Physical Sciences 
Research Council (EPSRC) (EP/P000940/1); the National Natural Science Foundation of China (NSFC) (61705072).

\section{References}

[1]. J. Leuthold, C. Koos, W. Freude, Nonlinear silicon photonics, Nature Photon. 4 (2010) 535-544.

[2]. R. M. Osgood, N. C. Panoiu, J. I. Dadap, X. Liu, X. Chen, I-W. Hsieh, E. Dulkeith, W. M. J. Green, Y. A. Vlasov, Engineering nonlinearities in nanoscale optical systems: physics and applications in dispersion-engineered silicon nanophotonic wires, Adv. Opt. Photonics 1 (2009)162-235.

[3]. M. A. Foster, A. C. Turner, J. E. Sharping, B. S. Schmidt, M. Lipson, A. L. Gaeta, Broad-band optical parametric gain on a silicon photonic chip, Nature 441(2006) 960963.

[4]. X. Liu, B. Kuyken, G. Roelkens, R. Baets, R. M. Osgood, W. M. J. Green, Bridging the mid-infrared-to-telecom gap with silicon nanophotonic spectral translation, Nature Photon. 6 (2012) 667-671.

[5]. Q. Lin, O. J. Painter, G. P. Agrawal, Nonlinear optical phenomena in silicon waveguides: Modeling and applications, Opt. Express 15 (2007) 16604-16644.

[6]. J. Ballato, T. Hawkins, P. Foy, R. Stolen, B. Kokuoz, M. Ellison, C. McMillen, J. Reppert, A. M. Rao, M. Daw, S. Sharma, R. Shori, O. Stafsudd, R. R. Rice, D. R. Powers, Silicon optical fiber, Opt. Express 16 (2008) 18675-18683.

[7]. E. F. Nordstrand, A. N. Dibbs, A. J. Eraker, U. J. Gibson, Alkaline oxide interface modifiers for silicon fiber production, Opt. Mater. Express 3 (2013) 651-657. 
[8]. F. H. Suhailin, L. Shen, N. Healy, L. Xiao, M. Jones, T. Hawkins, J. Ballato, U. J. Gibson, A. C. Peacock, Tapered polysilicon core fibers for nonlinear photonics, Opt. Lett. 41 (2016) 1360-1363.

[9]. Y. Franz, A. F. J. Runge, H. Ren, N. Healy, K. Ignatyev, M. Jones, T. Hawkins, J. Ballato, U. J. Gibson, A. C. Peacock, Material properties of tapered crystalline silicon core fibers, Opt. Mater. Express 7 (2017) 2055-2061.

[10]. H. Ren, O. Aktas, Y. Franz, A. F. J. Runge, T. Hawkins, J. Ballato, U. J. Gibson, A. C. Peacock, Tapered silicon core fibers with nano-spikes for optical coupling via spliced silica fibers, Opt. Express 25 (2017) 24157-24163.

[11]. A. C. Peacock, J. Campling, A. F. J. Runge, H. Ren, L. Shen, O. Aktas, P. Horak, N. Healy, U. J. Gibson, J. Ballato, Wavelength Conversion and Supercontinuum Generation in Silicon Optical Fibers, IEEE Journal of Selected Topics in Quantum Electronics 24 (2018) 5100309.

[12]. Y. Ye, L. Xiao, S. Dong, A. C. Peacock, Analysis of Coupling Losses for All-Fiber Integration of Subwavelength Core Hybrid Optical Fibers, IEEE Photonics Journal 10 (2018) 7104212.

[13]. M. Huang, H. Ren, O. Aktas, L. Shen, J. Wang, T. W. Hawkins, J. Ballato, U. J. Gibson, A. C. Peacock, Fiber Integrated Wavelength Converter Based on a Silicon Core Fiber with a Nano-spike Coupler, IEEE Photonics Technology Letters 31 (2019) 15611564.

[14]. P. Mehta, N. Healy, N. F. Baril, P. J. A. Sazio, J. V. Badding, A. C. Peacock, Nonlinear transmission properties of hydrogenated amorphous silicon core optical fibers, Opt. Express 18 (2010) 16826-16831. 
[15]. P. Mehta, N. Healy, T. D. Day, J. R. Sparks, P. J. A. Sazio, J. V. Badding, A. C. Peacock, All-optical modulation using two-photon absorption in silicon core optical fibers, Opt. Express 19 (2011) 19078-19083.

[16]. P. Mehta, N. Healy, T. D. Day, J. V. Badding, A. C. Peacock, Ultrafast wavelength conversion via cross-phase modulation in hydrogenated amorphous silicon optical fibers, Opt. Express 20 (2012) 26110-26116.

[17]. L. Shen, N. Healy, L. Xu, H. Y. Cheng, T. D. Day, J. H. V. Price, J. V. Badding, A.

C. Peacock, "Four-wave mixing and octave-spanning supercontinuum generation in a small core hydrogenated amorphous silicon fiber pumped in the mid-infrared," Opt. Lett. 39 (2014) 5721-5724.

[18]. D. Wu, L. Shen, H. Ren, J. Campling, T. W. Hawkins, J. Ballato, U. J. Gibson, A. C. Peacock, Net optical parametric gain in a submicron silicon core fiber pumped in the telecom band, APL Photonics 4 (2019) 086102.

[19]. A. C. Peacock, N. Healy, Semiconductor optical fibres for infrared applications: A review, Semicond. Sci. Technol. 31 (2016), 103004.

[20]. P. J. A. Sazio, A. Amezcua-Correa, C. E. Finlayson, J. R. Hayes, T. J. Scheidemantel, N. F. Baril, B. R. Jackson, D.-J. Won, F. Zhang, E. R. Margine, V. Gopalan, V. H. Crespi, J. V. Badding, Microstructured optical fibers as high-pressure microfluidic reactors, Science 311 (2006), 1583-1586.

[21]. J. Ballato, A. C. Peacock, Perspective: Molten core optical fiber fabrication-A route to new materials and applications, APL Photonics 3 (2018) 120903.

[22]. T.A. Birks, Y.W. Li, The shape of fiber tapers, Journal of Lightwave Technology 10 (1992) 432-438. 
[23]. N. Healy, J. R. Sparks, P. J. A. Sazio, J. V. Badding, A. C. Peacock, Tapered silicon optical fibers, Opt. Express 18 (2010) 7596-7601.

[24]. L. Yin, G. Agrawal, " Impact of two-photon absorption on self-phase modulation in silicon waveguides, Opt. Lett. 32 (2007) 2031-2033.

[25]. H. Ren, L. Shen, T. W. Hawkins, J. Ballato, U. J. Gibson, A. C. Peacock, Nonlinear optical properties of polycrystalline silicon core fibers from telecom wavelengths into the mid-infrared spectral region, Opt. Mater. Express 9 (2019) 1271-1279.

[26]. X. P. Liu, J. B. Driscoll, J. I. Dadap, R. M. Osgood, S. Assefa, Y. A. Vlasov, W. M. J. Green, Self-phase modulation and nonlinear loss in silicon nanophotonic wires near the mid-IR two-photon absorption edge, Opt. Express 19 (2011) 7778-7789.

[27]. A. D. Bristow, N. Rotenberg, H. M. van Driel, Two-photon absorption and Kerr coefficients of silicon for 850-2200nm, Appl. Phys. Lett. 90 (2007) 191104.

[28]. Q. Lin, J. Zhang, G. Piredda, R. W. Boyd, P. M. Fauchet, G. P. Agrawal, Dispersion of silicon nonlinearities in the near infrared region, Appl. Phys. Lett. 91 (2007) 02111. [29]. H. Garcia, R. Kalyanaraman, Phonon-assisted two-photon absorption in the presence of a dc-field: the nonlinear Franz-Keldysh effect in indirect gap semiconductors, J. Phys. B: At. Mol. Opt. Phys. 39 (2006) 2737-2746.

[30]. N. Hon, R. Soref, B. Jalali, The third-order nonlinear optical coefficients of Si, Ge, and $\mathrm{Si}_{1-\mathrm{x}} \mathrm{Ge}_{\mathrm{x}}$ in the midwave and longwave infrared, J. Appl. Phys. 110 (2011) 011301. [31]. F. Gholami, S. Zlatanovic, A. Simic, L. Liu, D. Borlaug, N. Alic, M. Nezhad, Y. Fainman, S. Radic, Third-order nonlinearity in silicon beyond 2350 nm, Appl. Phys. Lett. 99 (2011) 081102. 
[32]. R. J. Kruhlak, G. K. Wong, J. S. Chen, S. G. Murdoch, R. Leonhardt, J. D. Harvey, N. Y. Joly, J. C. Knight, Polarization modulation instability in photonic crystal fibers, Opt. Lett. 31 (2006) 1379-1381.

[33]. A. C. Turner, C. Manolatou, B. S. Schmidt, M. Lipson, M. A. Foster, J. E. Sharping, A. L. Gaeta, Tailored anomalous group-velocity dispersion in silicon channel waveguides, Opt. Express 14 (2006) 4357-4362.

[34]. K. J. A. Ooi, D. K. T. Ng, T. Wang, A. K. L. Chee, S. K. Ng, Q. Wang, L. K. Ang, A. M. Agarwal, L. C. Kimerling, D. T. H. Tan, Pushing the limits of CMOS optical parametric amplifiers with USRN: $\mathrm{Si}_{7} \mathrm{~N}_{3}$ above the two-photon absorption edge, Nat. Commun. 8 (2017) 13878.

[35]. X. Liu, B. Kuyken, G. Roelkens, R. Baets, R. M. Osgood, Jr., W. M. J. Green, Bridging the mid-infrared-to-telecom gap with silicon nanophotonic spectral translation, Nat. Photonics 6 (2012) 667-671.

[36]. A. M. Heidt, Pulse preserving flat-top supercontinuum generation in all-normal dispersion photonic crystal fibers, J. Opt. Soc. Am. B 27 (2010) 550-559.

[37]. H. Hu, X. Zhang, W. Li, N. Dutta, Simulation of octave spanning mid-infrared supercontinuum generation in dispersion-varying planar waveguides, Appl. Opt, 54 (2015), 3448-3454.

[38]. N. Singh, D. Vermulen, A. Ruocco, N. Li, E. Ippen, F. X. Kärtner, M. R. Watts, Supercontinuum generation in varying dispersion and birefringent silicon waveguide, Opt. Express 27 (2019) 31698-31712.

[39]. C. Ciret. S.-P. Gorza, Generation of ultra-broadband coherent supercontinua in tapered and dispersion-managed silicon nanophotonic waveguides, Journal of the Optical Society of America B 34 (2017) 1156-1162. 
[40]. H. Ren, L. Shen, J. Campling, A. F. J. Runge, O. Aktas, T. Hawkins, P. Horak, J. Ballato, U. Gibson, A. C. Peacock, Octave-spanning supercontinuum generation in a dispersion managed tapered crystalline silicon core fiber. Proceedings of Conference on Lasers and Electro-Optics. San Jose, CA, USA: OSA, 2018

[41]. H. Ren, L. Shen, A. F. J. Runge, T. W. Hawkins, J. Ballato, U. J. Gibson, A. C. Peacock, Low-loss silicon core fibre platform for broadband nonlinear photonics in the mid-infrared, Light Sci Appl, (2019) in press.

[42]. V. R. Almeida, R. R. Panepucci, M. Lipson, Nanotaper for compact mode conversion, Opt. Lett. 28 (2003) 1302-1304. 